\title{
Mudanças temporais na abundância e diversidade da fauna de peixes do infralitoral raso de uma praia, sul do Brasil
}

\author{
Rodrigo S. Godefroid; Henry L. Spach; Cesar Santos; Guilherme MacLaren \& Roberto Schwarz Jr. \\ Centro de Estudos do Mar, Universidade Federal do Paraná, Caixa Postal 50002, 83255-000, Pontal do Paraná, PR, Brasil.
}

\begin{abstract}
Temporal changes in the abundance and diversity of the fish fauna in the shallow infralittoral of a beach, southern Brazil. Abundance and diversity of the fish fauna in the shallow infralittoral region of Atami Beach, Paraná, Brazil, were studied through monthly collections between July 1998 and June 1999. A total of 7553 fishes from 70 species and 26 families, mainly juvenile forms, was captured in the area. The mean captures in number of species, number of fish and biomass were larger from the end of spring to the middle of autumn; seasonal tendency in richness, diversity (number) and equitability indices were not observed. The largest mean dissimilarity in the composition of the ichthyofauna occurred between June and May in comparison to the period between July and October. The species that contributed most to this dissimilarity were Selene setapinnis (Mitchill, 1815), Conodon nobilis (Linnaeus, 1758) and Stellifer rastrifer (Jordan, 1889). Three great groups reflecting patterns of temporal occurrence of the most abundant species were identified.
\end{abstract}

KEYWORDS. Demersal fish, temporal variation, abundance, diversity, Brazil.

\section{INTRODUÇÃO}

A estrutura da ictiofauna tem sido estudada em ecossistemas de águas continentais, recifes de corais e em regiões marinhas costeiras, como baías e estuários (BlABER et al., 1989; YoKLAVICH et al., 1991; JACKSON \& JONES, 1999). Em função da atividade pesqueira, vários trabalhos têm analisado a estrutura da comunidade de peixes demersais da plataforma e talude continental (Bergstad et al., 1999; Kallianotis et al., 2000).

$\mathrm{Na}$ plataforma continental do sudeste e sul do Brasil, os estudos sobre a ictiofauna demersal objetivaram identificar os padrões de distribuição e abundância e as possíveis influências dos parâmetros ambientais sobre a estrutura dessas comunidades de peixes (HAImovici et al., 1996; Rocha \& Rossi-Wongtschowski, 1998; Muto et al., 2000). No litoral paranaense, a composição e a variação espaço-temporal da ictiofauna demersal foram estudadas principalmente nas áreas estuarinas (CHAVES \& CorrêA, 1998; Chaves \& Bouchereau, 1999).

O objetivo é identificar a composição e o padrão de variação temporal da assembléia de peixes no infralitoral raso de uma praia arenosa exposta, próxima à barra de acesso sul da baía de Paranaguá.

\section{MATERIAL E MÉTODOS}

A área de estudo, infralitoral raso da praia Balneário Atami (fig.1), situa-se na região centro-norte do litoral do

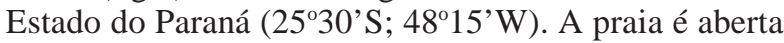
para o oceano, sendo a primeira a se desenvolver sem a influência dos bancos submersos que constituem a barra sul da entrada da baía de Paranaguá. Com o objetivo de caracterizar a comunidade de peixes e suas variações temporais, os peixes do infralitoral raso da praia Balneário Atami foram coletados mensalmente entre julho de 1998 e junho de 1999. Para obtenção das amostras, utilizou-se canoa de madeira de $7 \mathrm{~m}$ movida com motor de popa com
$16 \mathrm{Hp}$. Foram realizados arrastos de 40 minutos, a uma velocidade de 1,5 nós, paralelos à costa e em profundidades entre 8 e $12 \mathrm{~m}$, com rede do tipo porta com $8 \mathrm{~m}$ de boca, $6,5 \mathrm{~m}$ de ensacador, $1 \mathrm{~cm}$ de malha entre nós adjacentes e duas portas de madeira de $70 \mathrm{~cm} \mathrm{x} 47 \mathrm{~cm}$, com $8 \mathrm{~kg}$ cada. Em laboratório, após a identificação dos indivíduos, foram obtidos: peso (g), comprimento padrão e total $(\mathrm{Cp}$ e $\mathrm{Ct}$, $\mathrm{mm}$ ) e, quando possível, determinado o sexo. Os peixes analisados durante o estudo estão depositados no Laboratório de Biologia de Peixes do Centro de Estudos do Mar, Universidade Federal do Paraná.

Para análise dos dados, as amostras foram agrupadas por mês de coleta e as variações temporais da composição e da abundância da comunidade avaliadas através da comparação das capturas médias em número de espécies, número de peixes e peso total e dos índices de estrutura da comunidade: riqueza de espécies de Margalef, diversidade de Shannon-Wiener (captura em

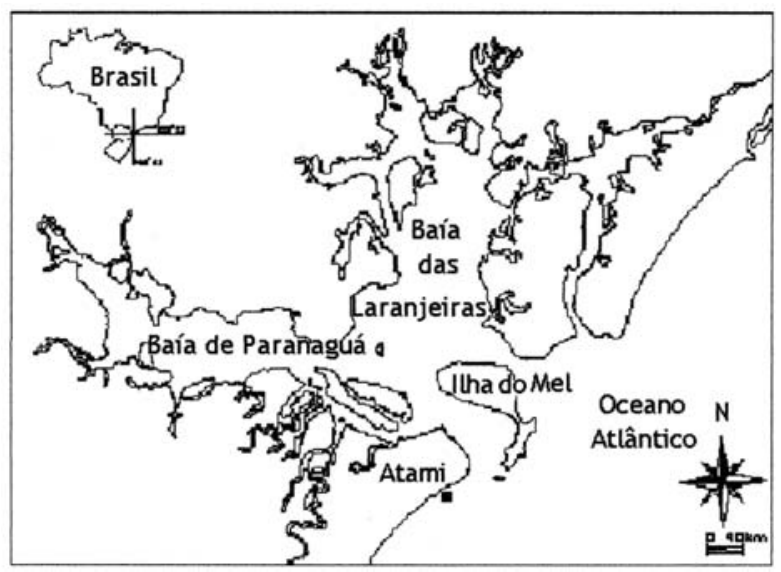

Fig. 1. Litoral paranaense, com a localização do ponto de amostragem (ם) na praia Balneário de Atami. 
número e peso), e de eqüitatividade de Pielou (PIElou, 1969). Estas médias foram comparadas através da análise de variância univariada (ANOVA), tendo como fator fixo o mês de coleta, aplicada aos dados raiz quarta transformados e testados quanto à homogeneidade de variância (teste de Bartelett) e normalidade das distribuições (prova de Kolmogorov-Smirnov). Quando o resultado indicava diferença significativa $(\mathrm{p}<0,01 \mathrm{e}$ $\mathrm{p}<0,05)$, aplicou-se o teste a posteriori de Mínimas Diferenças Significativas (LSD), para identificar quais médias eram diferentes. Nos casos em que um dos pressupostos da ANOVA não foi atendido, aplicou-se o teste não paramétrico de Kruskal-Wallis (CONOvER, 1990; SoKAl \& RoHLF, 1995).

Com o objetivo de identificar as associações, aplicou-se aos dados de densidade raiz quarta transformados das espécies mais abundantes (pelo menos $1 \%$ da captura total ou presença em no mínimo 6 meses de coleta), a Análise de Agrupamento modo-R (Cluster, método inverso), a qual procura agrupar as espécies tendo como atributos a sua abundância nos meses do ano. A estrutura temporal das associações foi identificada, utilizando-se a Análise de Agrupamento modo-Q (Cluster, método normal), tendo como atributo a abundância das espécies mais representativas. A similaridade entre os atributos foi calculada através do coeficiente de similaridade de Bray-Curtis e o método de agrupamento, pela média simples dos seus valores de similaridade (UPGMA) (LuDWIG \& REYNOLDS, 1988). Como nem sempre existem entidades discretas na natureza, aplicou-se aos mesmos dados submetidos a Análise de Cluster, a técnica de ordenação MDS (Non-Metric Multidimensional Scaling), que está baseada na existência de um "continuum" na natureza. Os eixos de ordenação representariam gradientes biológicos ao longo dos quais as espécies ou meses se distribuiriam (GAUCH, 1982).

Considerando-se como fator os grupos de meses definidos pelo Cluster, aplicou-se a ANOVA, do mesmo modo como descrito anteriormente, para avaliar as diferenças no número de espécies e peixes, peso, riqueza de Margalef, diversidade (número e peso) e eqüitatividade. A rotina analítica SIMPER (similaridade de percentagens) foi utilizada para identificar as espécies mais responsáveis (espécies mais comuns) pela similaridade dentro de cada grupo definido pelo Cluster (modo Q) e para dissimilaridade entre esses grupos (espécies mais discriminantes) (ClARKE \& WARWICK, 1994). Todos os procedimentos analíticos foram realizados através dos programas estatísticos Statistica, versão 4.0 e Primer, versão 5.1.2. As estações do ano foram definidas da seguinte forma: setembro a novembro, primavera; dezembro a fevereiro, verão; março a maio, outono; junho a agosto, inverno.

\section{RESULTADOS}

Um total de 7553 peixes e 70 espécies pertencentes a 26 famílias foram reportados nas capturas mensais realizadas durante o período de um ano (tab. I). As formas juvenis imaturas foram dominantes, representando 79,6\% das capturas. Entre os adultos predominaram os machos $(64,5 \%)$ sobre as fêmeas $(35,5 \%)$. Das espécies presentes nas amostras, 36 ocorreram como indivíduos juvenis e adultos, 30 espécies só por juvenis e 4 espécies só por adultos. Das famílias coletadas, Sciaenidae (18 espécies) e Carangidae (9 espécies) apresentaram o maior número de espécies. Dominou nas amostragens um pequeno número de espécies. Estas, embora sejam comuns nos arrastos de pesca comercial, não são economicamente importantes (Selene setapinnis, Conodon nobilis, Orthopristis ruber, Larimus breviceps, Paralonchurus brasiliensis, Stellifer brasiliensis, Stellifer rastrifer). Cada uma destas espécies representou mais de 2,5\% do número total de capturas. Stellifer rastrifer $(29,9 \%)$ foi a espécie mais abundante no infralitoral do Balneário de Atami, mas não esteve presente na área em junho. Apenas três espécies (Pellona harroweri, L. breviceps e Lagocephalus laevigatus) estiveram representadas durante todo o período de estudo. A contribuição das quatro espécies mais numerosas (C. nobilis, L. breviceps, P. brasiliensis e $S$. rastrifer) atingiu $59 \%$ do total de peixes, porém chegou a $70 \%$ das capturas em peso, indicando que peixes com maior comprimento influenciaram a estrutura em tamanho (Cp: 100 a 154 mm). A composição específica em peso apresentou um padrão um pouco diferente. $\mathrm{O}$ valor do peso total foi afetado pela presença de indivíduos com tamanhos maiores de $S$. rastrifer (Cp: 80 a 104,5 mm), P. brasiliensis (Cp: 100 a 154 $\mathrm{mm}$ ) e de L. breviceps (Cp: 100 a 136,5 mm). Por exemplo, S. rastrifer, que foi a espécie com a maior contribuição numérica $(29,9 \%)$, também foi a principal contribuinte em termos de peso das capturas, com 44,6\%; P. brasiliensis, a terceira maior contribuinte em número de peixes capturados $(8,7 \%)$, com relação ao peso das capturas acabou ocupando a segunda posição, com uma contribuição de $10,5 \%$ e L. breviceps, que foi a segunda espécie numericamente dominante $(14,0 \%)$ e apresentou a terceira maior contribuição em peso $(6,8 \%)$ (tab. II).

Uma tendência sazonal foi observada no número de espécies, com valores maiores do final da primavera até a metade do outono, valores intermediários no inverno e menores em maio e nos dois primeiros meses da primavera (fig. 2). Com exceção de outubro, todos os demais meses apresentaram médias de capturas em número de espécies significativamente maiores do que as observadas em maio e setembro, meses com menor número médio de espécies nas amostras (tab. III). Um maior número de peixes foi capturado entre o final da primavera e metade do outono, sendo menores a partir de maio até a metade da primavera (fig. 2), com as médias estatisticamente diferentes entre os dois períodos (tab. III). Em peso, a captura foi significativamente maior em novembro e menor em maio, com o período da metade da primavera até a metade do outono, exceção de fevereiro, apresentando as maiores capturas médias em comparação como o intervalo entre maio e setembro (fig. 2).

Nenhuma tendência estacional foi observada na riqueza e diversidade (número) das capturas (fig. 2; tab. III). A diversidade ( $\mathrm{H}^{\prime}$ peso) apresentou valores maiores do início do verão até a metade do outono, valores médios em novembro e de maio até o final do inverno, com os menores valores ocorrendo em setembro e outubro (fig. 2). As médias não diferiram significativamente entre o verão e o inverno. As diferenças estatísticas estiveram 
Tabela I. Espécies de peixes, número de exemplares capturados na zona rasa do infralitoral da praia Balneário de Atami, Paraná, entre julho de 1998 e junho de 1999 e porcentagem do total capturado (PTC).

\begin{tabular}{|c|c|c|c|c|c|c|c|c|c|c|c|c|c|}
\hline Espécies & Jul & Ago & Set & Out & Nov & Dez & Jan & Fev & Mar & Abr & Mai & Jun & $\begin{aligned} \text { PTC } \\
(\%)\end{aligned}$ \\
\hline Narcine brasiliensis (Olfers, 1831) & 0 & 2 & 0 & 0 & 1 & 0 & 0 & 0 & 2 & 1 & 1 & 1 & 0,10 \\
\hline Cynoponticus savanna (Bancroft, 1831) & 0 & 0 & 0 & 0 & 0 & 0 & 0 & 1 & 0 & 1 & 0 & 0 & 0,03 \\
\hline Anchoa lyolepis (Evermann \& Marsh, 1900) & 0 & 0 & 0 & 0 & 0 & 0 & 0 & 0 & 0 & 1 & 0 & 0 & 0,01 \\
\hline Anchoa tricolor (Agassiz, 1829) & 0 & 0 & 0 & 0 & 0 & 0 & 2 & 1 & 2 & 0 & 0 & 0 & 0,07 \\
\hline Cetengraulis edentulus (Cuvier, 1829) & 1 & 0 & 0 & 0 & 49 & 0 & 0 & 0 & 0 & 0 & 0 & 0 & 0,66 \\
\hline Lycengraulis grossidens (Agassiz, 1829) & 0 & 0 & 0 & 0 & 29 & 0 & 2 & 1 & 0 & 0 & 0 & 0 & 0,42 \\
\hline Chirocentrodon bleekerianus (Poey, 1867) & 3 & 1 & 0 & 0 & 5 & 41 & 0 & 4 & 5 & 6 & 0 & 0 & 0,86 \\
\hline Harengula clupeola (Cuvier, 1829) & 0 & 0 & 0 & 0 & 1 & 0 & 0 & 0 & 1 & 0 & 0 & 0 & 0,03 \\
\hline Pellona harroweri (Fowler, 1917) & 8 & 7 & 2 & 8 & 37 & 6 & 18 & 2 & 1 & 3 & 2 & 15 & 1,44 \\
\hline Sardinella brasiliensis (Steindachner, 1879) & 0 & 3 & 0 & 0 & 0 & 0 & 6 & 0 & 0 & 0 & 0 & 0 & 0,12 \\
\hline Genidens genidens (Cuvier, 1829) & 3 & 1 & 1 & 0 & 23 & 5 & 130 & 1 & 0 & 2 & 0 & 0 & 2,20 \\
\hline Urophycis brasiliensis (Kaup, 1858) & 8 & 2 & 0 & 0 & 0 & 0 & 0 & 0 & 0 & 0 & 0 & 0 & 0,13 \\
\hline Porichthys porosissimus (Cuvier, 1829) & 0 & 0 & 0 & 1 & 3 & 0 & 0 & 0 & 0 & 0 & 0 & 0 & 0,05 \\
\hline Hemiramphus brasiliensis (Linnaeus, 1758) & 0 & 0 & 0 & 0 & 0 & 0 & 0 & 0 & 7 & 0 & 0 & 0 & 0,09 \\
\hline Hyporhamphus unifasciatus (Ranzani, 1842) & 0 & 0 & 0 & 0 & 0 & 0 & 0 & 0 & 1 & 0 & 0 & 0 & 0,01 \\
\hline Prionotus punctatus (Bloch, 1793) & 0 & 1 & 0 & 1 & 0 & 1 & 16 & 0 & 0 & 0 & 0 & 0 & 0,25 \\
\hline Pomatomus saltatrix (Linnaeus, 1766) & 1 & 0 & 0 & 0 & 0 & 0 & 0 & 0 & 0 & 0 & 0 & 0 & 0,01 \\
\hline Carangoides bartholomaei (Cuvier, 1833) & 0 & 0 & 0 & 0 & 0 & 0 & 0 & 0 & 0 & 2 & 0 & 0 & 0,03 \\
\hline Caranx ruber (Bloch, 1793) & 0 & 0 & 0 & 0 & 0 & 3 & 8 & 100 & 16 & 5 & 0 & 7 & 1,84 \\
\hline Chloroscombrus chrysurus (Linnaeus, 1766) & 0 & 0 & 0 & 0 & 0 & 0 & 0 & 0 & 26 & 0 & 0 & 0 & 0,34 \\
\hline Oligoplites saurus (Bloch \& Schneider, 1801) & 0 & 0 & 0 & 0 & 1 & 4 & 0 & 0 & 0 & 2 & 0 & 0 & 0,09 \\
\hline Selene setapinnis (Mitchill, 1815) & 1 & 0 & 0 & 0 & 150 & 29 & 18 & 6 & 1 & 0 & 3 & 155 & 4,81 \\
\hline Selene vomer (Linnaeus, 1758) & 0 & 0 & 0 & 0 & 2 & 13 & 0 & 7 & 1 & 1 & 1 & 8 & 0,44 \\
\hline Trachinotus carolinus (Linnaeus, 1766) & 0 & 0 & 0 & 0 & 0 & 0 & 2 & 0 & 0 & 0 & 0 & 0 & 0,03 \\
\hline Trachinotus marginatus Cuvier, 1832 & 0 & 0 & 0 & 0 & 0 & 0 & 0 & 0 & 1 & 0 & 0 & 0 & 0,01 \\
\hline Uraspis secunda (Poey, 1860) & 0 & 0 & 0 & 0 & 0 & 0 & 2 & 34 & 0 & 0 & 0 & 0 & 0,48 \\
\hline Diapterus rhombeus (Cuvier, 1829) & 0 & 0 & 0 & 0 & 0 & 1 & 0 & 0 & 0 & 0 & 0 & 0 & 0,01 \\
\hline Anisotremus surinamensis (Bloch, 1791) & 0 & 0 & 0 & 2 & 1 & 4 & 0 & 61 & 4 & 4 & 0 & 3 & 1,05 \\
\hline Conodon nobilis (Linnaeus, 1758) & 0 & 0 & 0 & 0 & 0 & 0 & 14 & 77 & 203 & 160 & 22 & 17 & 6,53 \\
\hline Haemulon steindachneri (Jordan \& Gilbert, 1882) & 0 & 1 & 0 & 0 & 0 & 0 & 0 & 0 & 0 & 0 & 0 & 0 & 0,01 \\
\hline Orthopristis ruber (Cuvier, 1830) & 0 & 0 & 0 & 0 & 0 & 0 & 0 & 0 & 219 & 0 & 0 & 0 & 2,90 \\
\hline Pomadasys corvinaeformis (Steindachner, 1868) & 0 & 0 & 0 & 0 & 2 & 0 & 87 & 5 & 0 & 0 & 0 & 0 & 1,24 \\
\hline Polydactylus oligodon (Günther, 1860) & 0 & 0 & 0 & 0 & 0 & 0 & 0 & 0 & 1 & 0 & 0 & 1 & 0,03 \\
\hline Polydactylus virginicus (Linnaeus, 1758) & 0 & 0 & 0 & 0 & 0 & 4 & 6 & 1 & 7 & 1 & 0 & 0 & 0,25 \\
\hline Bairdiella ronchus (Cuvier, 1830) & 1 & 0 & 0 & 0 & 0 & 0 & 0 & 0 & 19 & 7 & 0 & 0 & 0,36 \\
\hline Cynoscion jamaicensis (Vaillant \& Bocourt, 1883) & 0 & 0 & 0 & 0 & 0 & 0 & 0 & 0 & 0 & 12 & 0 & 0 & 0,16 \\
\hline Cynoscion leiarchus (Cuvier, 1830) & 0 & 0 & 0 & 0 & 0 & 22 & 20 & 4 & 3 & 5 & 0 & 0 & 0,71 \\
\hline Cynoscion microlepidotus (Cuvier, 1830) & 0 & 2 & 0 & 0 & 0 & 35 & 0 & 1 & 1 & 1 & 5 & 40 & 1,12 \\
\hline Isopisthus parvipinnis (Cuvier, 1830) & 0 & 2 & 0 & 3 & 27 & 0 & 6 & 1 & 6 & 8 & 11 & 21 & 1,12 \\
\hline Larimus breviceps Cuvier, 1830 & 1 & 14 & 7 & 2 & 18 & 41 & 352 & 308 & 105 & 196 & 4 & 10 & 14,01 \\
\hline Macrodon ancylodon (Bloch \& Schneider, 1801) & 1 & 0 & 0 & 0 & 0 & 0 & 0 & 0 & 0 & 0 & 0 & 0 & 0,01 \\
\hline Menticirrhus americanus (Linnaeus, 1758) & 0 & 0 & 0 & 2 & 6 & 4 & 134 & 41 & 11 & 16 & 0 & 1 & 2,85 \\
\hline Menticirrhus littoralis (Holbrook, 1860) & 2 & 0 & 0 & 0 & 5 & 70 & 0 & 26 & 21 & 2 & 0 & 1 & 1,68 \\
\hline Nebris microps Cuvier, 1830 & 0 & 0 & 0 & 0 & 0 & 0 & o & 0 & 1 & 1 & 0 & 0 & 0,03 \\
\hline Ophioscion punctatissimus Meek \& Hildebrand, 1925 & 0 & 0 & 0 & 1 & 0 & 2 & 12 & 0 & 0 & 0 & 0 & 0 & 0,25 \\
\hline Paralonchurus brasiliensis (Steindachner, 1875) & 0 & 30 & 3 & 75 & 162 & 158 & 74 & 65 & 27 & 42 & 3 & 18 & 8,70 \\
\hline Pogonias cromis (Linnaeus, 1766) & 20 & 0 & 0 & 0 & 0 & 0 & 0 & 0 & 0 & 0 & 0 & 0 & 0,26 \\
\hline Stellifer brasiliensis (Schultz, 1945) & 0 & 0 & 0 & 9 & 31 & 46 & 154 & 25 & 34 & 66 & 3 & 1 & 4,88 \\
\hline Stellifer stellifer (Bloch, 1790) & 1 & 34 & 0 & 1 & 13 & 13 & 2 & 3 & 1 & 0 & 2 & 0 & 0,93 \\
\hline Stellifer rastrifer (Jordan, 1889) & 81 & 68 & 44 & 208 & 1065 & 108 & 124 & 86 & 180 & 285 & 9 & 0 & 29,89 \\
\hline Stellifer sp. & 11 & 0 & 1 & 5 & 83 & 0 & 0 & 11 & 0 & 0 & 0 & 0 & 1,47 \\
\hline Umbrina canosai Berg, 1895 & 0 & 1 & 0 & 0 & 0 & 2 & 0 & 2 & 0 & 0 & 0 & 0 & 0,07 \\
\hline Pseudopercis semifasciata (Cuvier, 1829) & 0 & 0 & 0 & 0 & 0 & 0 & 0 & 0 & 0 & 0 & 1 & 0 & 0,01 \\
\hline Chaetodipterus faber (Broussonet, 1782) & 0 & 0 & 0 & 0 & 0 & 0 & 0 & 0 & 2 & 6 & 0 & 0 & 0,11 \\
\hline Sphyraena guachancho Cuvier, 1829 & 0 & 0 & 0 & 0 & 0 & 0 & 0 & 0 & 1 & 2 & 0 & 0 & 0,04 \\
\hline Trichiurus lepturus Linnaeus, 1758 & 0 & 0 & 0 & 0 & 5 & 2 & 2 & 0 & 0 & 0 & 0 & 0 & 0,12 \\
\hline Peprilus paru (Linnaeus, 1758) & 1 & 4 & 0 & 1 & 13 & 0 & 0 & 0 & 0 & 0 & 14 & 37 & 0,93 \\
\hline Citharichthys spilopterus Günther, 1862 & 0 & 0 & 0 & 1 & 0 & 0 & 0 & 0 & 0 & 0 & 0 & 0 & 0,01 \\
\hline Etropus crossotus Jordan \& Gilbert, 1882 & 0 & 0 & 0 & 1 & 0 & 0 & 2 & 5 & 2 & 1 & 3 & 0 & 0,18 \\
\hline Paralichthys brasiliensis (Ranzani, 1842) & 0 & 0 & 0 & 0 & 0 & 0 & 20 & 0 & 0 & 0 & 0 & 0 & 0,26 \\
\hline Oncopterus darwinii Steindachner, 1874 & 3 & 2 & 0 & 0 & 0 & 0 & o & 0 & 0 & 0 & 0 & 0 & 0,07 \\
\hline Achirus lineatus (Linnaeus, 1758) & 1 & 13 & 1 & 0 & 1 & 0 & 0 & 0 & 0 & 3 & 0 & 0 & 0,25 \\
\hline Trinectes microphthalmus (Chabanaud, 1928) & 1 & 2 & 1 & 1 & 1 & 1 & 0 & 3 & 5 & 4 & 1 & 2 & 0,29 \\
\hline Trinectes paulistanus (Miranda-Ribeiro, 1915) & 5 & 4 & 3 & 0 & 3 & 0 & 20 & 8 & 1 & 7 & 0 & 0 & 0,67 \\
\hline Symphurus plagusia (Bloch \& Schneider, 1801) & 2 & 2 & 2 & 1 & 1 & 2 & 7 & 3 & 0 & 1 & 0 & 0 & 0,28 \\
\hline Symphurus tessellatus (Quoy \& Gaimard, 1824) & 0 & 0 & 0 & 0 & 0 & 0 & 7 & 0 & 0 & 0 & 0 & 0 & 0,09 \\
\hline Monacanthus ciliatus (Mitchill, 1818) & 0 & 1 & 0 & 0 & 0 & 0 & 0 & 0 & 0 & 0 & 0 & 0 & 0,01 \\
\hline Lagocephalus laevigatus (Linnaeus, 1766) & 3 & 14 & 4 & 2 & 41 & 25 & 14 & 5 & 1 & 1 & 1 & 1 & 1,48 \\
\hline Sphoeroides greeleyi (Gilbert, 1900) & 0 & 0 & 0 & 0 & 0 & 0 & 0 & 0 & 0 & 0 & 0 & 1 & 0,01 \\
\hline Sphoeroides testudineus (Linnaeus, 1758) & 0 & 0 & 0 & 3 & 3 & 2 & 0 & 0 & 1 & 0 & 0 & 0 & 0,12 \\
\hline
\end{tabular}


Tabela II. Espécies de peixes, peso dos exemplares capturados na zona rasa do infralitoral da praia Balneário de Atami, Paraná entre julho de 1998 e junho de 1999 e porcentagem do total capturado (PTC).

\begin{tabular}{|c|c|c|c|c|c|c|c|c|c|c|c|c|c|}
\hline Espécies & Jul & Ago & Set & Out & Nov & Dez & Jan & Fev & Mar & Abr & Mai & Jun & $\begin{array}{l}\text { PTC } \\
(\%)\end{array}$ \\
\hline Narcine brasiliensis & 0 & 108 & 0 & 0 & 28 & 0 & 0 & 0 & 22 & 13 & 9 & 26 & 0,3 \\
\hline Cynoponticus savanna & 0 & o & 0 & o & o & 0 & $\mathrm{O}$ & 74 & o & 43 & o & o & 0,1 \\
\hline Anchoa lyolepis & 0 & 0 & 0 & o & o & o & $\mathrm{O}$ & o & O & 2 & o & o & 0,0 \\
\hline Anchoa tricolor & 0 & o & 0 & o & 0 & o & 6 & 4 & 7 & o & o & o & 0,0 \\
\hline Cetengraulis edentulus & 54 & 0 & 0 & 0 & 1783 & o & 0 & 0 & 0 & o & o & 0 & 2,3 \\
\hline Lycengraulis grossidens & 0 & 0 & 0 & o & 1122 & o & 56 & 4 & 0 & 0 & o & o & 1,5 \\
\hline Chirocentrodon bleekerianus & 4 & 4 & 0 & o & 26 & 199 & 0 & 10 & 16 & 19 & 0 & 0 & 0,3 \\
\hline Harengula clupeola & o & 0 & 0 & o & 3 & o & o & 0 & 4 & 0 & o & 0 & 0,0 \\
\hline Pellona harroweri & 8 & 5 & 27 & 72 & 230 & 16 & 109 & 20 & 8 & 23 & 3 & 71 & 0,7 \\
\hline Sardinella brasiliensis & 0 & 47 & 0 & o & o & o & 186 & 0 & 0 & o & o & o & 0,3 \\
\hline Genidens genidens & 227 & 35 & 35 & o & 813 & 272 & 1036 & 5 & 0 & 23 & o & o & 3,0 \\
\hline Urophycis brasiliensis & 10 & 24 & o & o & o & o & o & o & 0 & o & o & o & 0,0 \\
\hline Porichthys porosissimus & o & 0 & 0 & 4 & 14 & o & 0 & o & 0 & o & o & o & 0,0 \\
\hline Hemiramphus brasiliensis & 0 & 0 & 0 & o & 0 & 0 & 0 & 0 & 47 & o & o & o & 0,1 \\
\hline Hyporhamphus unifasciatus & o & 0 & 0 & 0 & o & o & o & 0 & 38 & o & o & o & 0,0 \\
\hline Prionotus punctatus & o & 10 & 0 & 11 & o & 6 & 54 & o & 0 & o & o & o & 0,1 \\
\hline Pomatomus saltatrix & 3 & 0 & 0 & o & o & 0 & 0 & 0 & 0 & o & 0 & o & 0,0 \\
\hline Carangoides bartholomaei & 0 & 0 & 0 & 0 & $\mathrm{O}$ & 0 & 0 & $\mathrm{O}$ & $\mathrm{O}$ & 4 & 0 & 0 & 0,0 \\
\hline Caranx ruber & o & 0 & 0 & o & o & 7 & 16 & 85 & 47 & 14 & o & 13 & 0,2 \\
\hline Chloroscombrus chrysurus & o & 0 & 0 & o & o & o & 0 & o & 98 & 0 & o & 0 & 0,1 \\
\hline Oligoplites saurus & o & o & 0 & o & 4 & 35 & 0 & o & 0 & 6 & o & 0 & 0,1 \\
\hline Selene setapinnis & 3 & o & 0 & o & 333 & 44 & 46 & 6 & 1 & o & 6 & 359 & 1,0 \\
\hline Selene vomer & 0 & 0 & 0 & o & 2 & 16 & o & 10 & 1 & 0 & 6 & 25 & 0,1 \\
\hline Trachinotus carolinus & o & 0 & 0 & o & o & o & 287 & o & 0 & o & o & o & 0,4 \\
\hline Trachinotus marginatus & o & o & 0 & o & o & o & o & 0 & 36 & o & o & o & 0,0 \\
\hline Uraspis secunda & 0 & 0 & 0 & o & o & o & 44 & 88 & 0 & o & o & o & 0,2 \\
\hline Diapterus rhombeus & 0 & 0 & 0 & 0 & 0 & 32 & 0 & 0 & 0 & o & o & o & 0,0 \\
\hline Anisotremus surinamensis & 0 & 0 & 0 & 38 & 24 & 118 & 0 & 218 & 29 & 13 & o & 22 & 0,6 \\
\hline Conodon nobilis & 0 & 0 & 0 & o & 0 & o & 88 & 492 & 2076 & 1885 & 148 & 172 & 6,0 \\
\hline Haemulon steindachneri & o & 9 & 0 & o & o & o & o & o & o & o & o & o & 0,0 \\
\hline Orthopristis ruber & o & o & 0 & o & 0 & o & 0 & 0 & 377 & o & o & o & 0,5 \\
\hline Pomadasys corvinaeformis & o & o & 0 & o & 85 & o & 541 & 34 & o & o & o & 0 & 0,8 \\
\hline Polydactylus oligodon & 0 & 0 & 0 & o & 0 & o & 0 & 0 & 19 & o & o & 11 & 0,0 \\
\hline Polydactylus virginicus & 0 & 0 & 0 & 0 & o & 37 & 95 & 21 & 107 & 20 & o & 0 & 0,3 \\
\hline Bairdiella ronchus & 1 & 0 & 0 & 0 & 0 & o & 0 & 0 & 176 & 51 & o & o & 0,3 \\
\hline Cynoscion jamaicensis & 0 & 0 & 0 & 0 & $\mathrm{O}$ & 0 & 0 & 0 & o & 165 & o & o & 0,2 \\
\hline Cynoscion leiarchus & o & 0 & 0 & o & 0 & 103 & 99 & 22 & 37 & 30 & o & o & 0,4 \\
\hline Cynoscion microlepidotus & o & 8 & 0 & o & o & 121 & 0 & 4 & 5 & 6 & 31 & 231 & 0,5 \\
\hline Isopisthus parvipinnis & o & 7 & 0 & 126 & 458 & o & 58 & 18 & 67 & 209 & 43 & 362 & 1,7 \\
\hline Larimus breviceps & 17 & 74 & 16 & 36 & 793 & 74 & 1170 & 534 & 1119 & 1572 & 18 & 81 & 6,8 \\
\hline Macrodon ancylodon & 2 & 0 & 0 & o & o & o & o & o & o & o & o & 0 & 0,0 \\
\hline Menticirrhus americanus & 0 & 0 & 0 & 166 & 245 & 449 & 1144 & 473 & 199 & 228 & 0 & 13 & 3,6 \\
\hline Menticirrhus littoralis & 32 & 0 & 0 & o & 114 & 1322 & o & 324 & 183 & 23 & 0 & 0 & 2,5 \\
\hline Nebris microps & 0 & 0 & 0 & o & o & o & o & o & 35 & 21 & o & 0 & 0,1 \\
\hline Ophioscion punctatissimus & o & 0 & 0 & 49 & 0 & 24 & 126 & 0 & o & o & 0 & 0 & 0,2 \\
\hline Paralonchurus brasiliensis & o & 669 & 47 & 505 & 1328 & 1538 & 1097 & 953 & 510 & 949 & 109 & 855 & 10,5 \\
\hline Pogonias cromis & 457 & $\mathrm{O}$ & 0 & $\mathrm{O}$ & o & $\mathrm{O}$ & $\mathrm{O}$ & 0 & 0 & o & $\mathrm{o}$ & o & 0,6 \\
\hline Stellifer brasiliensis & o & o & 0 & 100 & 238 & 294 & 675 & 186 & 167 & 349 & 26 & 11 & 2,5 \\
\hline Stellifer stellifer & 4 & 312 & o & 19 & 218 & 136 & 45 & 35 & 19 & o & 13 & o & 1,0 \\
\hline Stellifer rastrifer & 1150 & 677 & 734 & 3663 & 22553 & 961 & 2286 & 497 & 1795 & 1833 & 56 & o & 44,6 \\
\hline Stellifer sp. & 68 & o & 1 & 14 & 808 & o & o & 93 & o & o & o & o & 1,2 \\
\hline Umbrina canosai & 0 & 1 & 0 & 0 & o & 4 & 0 & 2 & 0 & o & o & o & 0,0 \\
\hline Pseudopercis semifasciata & o & 0 & 0 & o & 0 & o & 0 & 0 & 0 & o & 9 & o & 0,0 \\
\hline Chaetodipterus faber & o & 0 & 0 & o & 0 & o & 0 & 0 & 3 & 101 & o & o & 0,1 \\
\hline Sphyraena guachancho & o & 0 & 0 & o & 0 & 0 & o & 0 & 10 & 36 & o & 0 & 0,1 \\
\hline Trichiurus lepturus & o & 0 & 0 & o & 5 & 6 & 96 & 0 & 0 & 0 & 0 & o & 0,1 \\
\hline Peprilus paru & 2 & 5 & 0 & 3 & 43 & o & 0 & 0 & 0 & 0 & 24 & 166 & 0,3 \\
\hline Citharichthys spilopterus & o & 0 & 0 & 21 & o & o & 0 & o & 0 & o & o & o & 0,0 \\
\hline Etropus crossotus & o & 0 & 0 & 21 & o & o & 6 & 23 & 12 & 5 & 9 & o & 0,1 \\
\hline Paralichthys brasiliensis & o & o & 0 & o & 0 & o & 173 & o & 0 & o & o & o & 0,2 \\
\hline Oncopterus darwinii & 12 & 18 & 0 & o & 0 & o & o & 0 & 0 & o & o & o & 0,0 \\
\hline Achirus lineatus & 21 & 76 & 2 & 0 & 4 & o & 0 & o & 0 & 57 & o & 0 & 0,2 \\
\hline Trinectes microphthalmus & 3 & 10 & 7 & 7 & 3 & 1 & 0 & 20 & 65 & 47 & 7 & 31 & 0,2 \\
\hline Trinectes paulistanus & 84 & 40 & 26 & 0 & 119 & o & 209 & 100 & 51 & 99 & o & 0 & 0,9 \\
\hline Symphurus plagusia & 78 & 12 & 13 & 6 & 7 & 7 & 36 & 18 & 0 & 5 & o & o & 0,2 \\
\hline Symphurus tessellatus & o & 0 & 0 & o & o & o & 36 & o & 0 & o & o & o & 0,0 \\
\hline Monacanthus ciliatus & o & 8 & o & 0 & o & o & o & o & o & 0 & o & o & 0,0 \\
\hline Lagocephalus laevigatus & 14 & 40 & 18 & 8 & 153 & 149 & 88 & 110 & 14 & 17 & 4 & 10 & 0,8 \\
\hline Sphoeroides greeleyi & o & 0 & 0 & 0 & o & o & o & o & 0 & 0 & o & 3 & 0,0 \\
\hline Sphoeroides testudineus & 0 & 0 & 0 & 181 & 213 & 193 & 0 & 0 & 162 & 0 & 0 & o & 0,9 \\
\hline
\end{tabular}


presentes entre os meses de setembro e outubro com relação ao verão e o inverno (tab. III). A eqüitatividade foi máxima em agosto e atingiu o menor valor em outubro, porém as variações mensais não permitiram a identificação de nenhum padrão sazonal (fig. 2, tab. III).
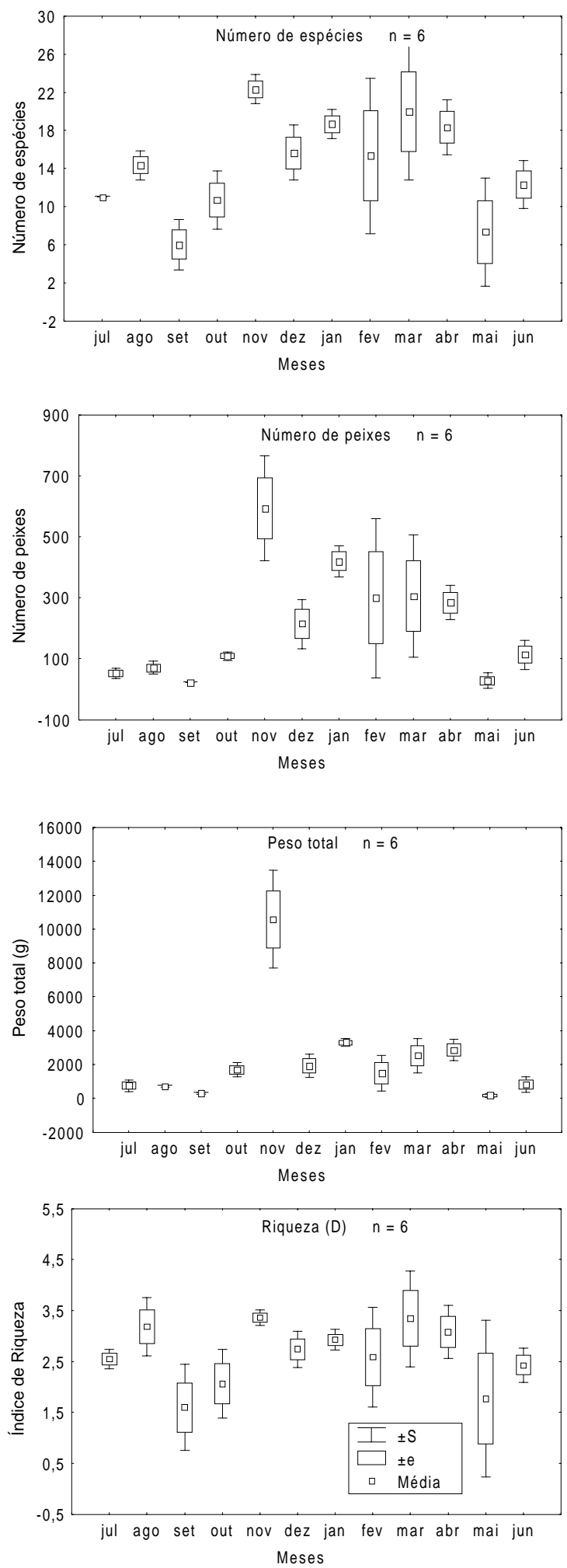

A análise de Cluster das amostras identificou um nível de similaridade de $59 \%$ entre três grupos de meses (fig. 3). O grupo A, contendo os meses de julho a outubro, é unido ao nível de similaridade de $60 \%$, e representa período com pequenas capturas médias em
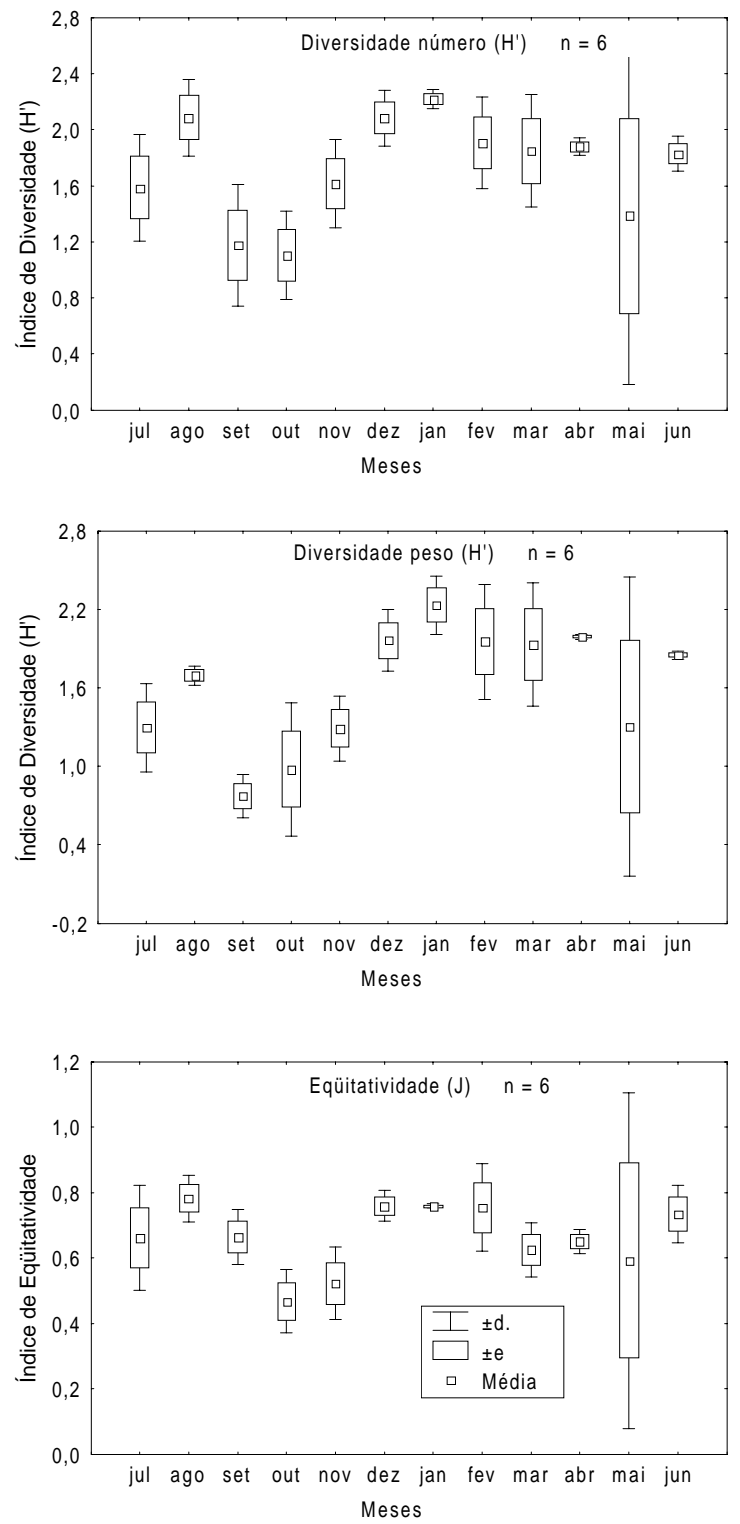

Fig. 2. Variação mensal na média do número de espécies, número de peixes, peso total e dos índices de riqueza de Margalef, diversidade de Shannon-Wiener e eqüitatividade de Pielou na zona rasa do infralitoral da praia Balneário de Atami, Paraná, entre julho de 1998 e junho de 1999. 
Tabela III. Resultado da ANOVA (F) e do teste de Kruskal-Wallis (H), avaliando o efeito do mês de coleta e dos grupos de meses definidos no Cluster, sobre a captura média em número de espécies, número de peixes, peso total, riqueza de espécies de Margalef, diversidade de Shannon-Wiener (número e peso) e eqüitatividade de Pielou, na zona rasa do infralitoral da praia Balneário de Atami, Paraná (NS, diferença não significativa; **, diferença significativa no nível de $\mathrm{p}<0,01$; *, diferença significativa no nível de $\mathrm{p}<0,05$ ).

\begin{tabular}{|c|c|c|c|c|c|c|c|c|c|}
\hline & \multicolumn{4}{|c|}{ MES } & \multicolumn{2}{|c|}{ GRUPO } & \multirow[b]{2}{*}{$\mathrm{H}$} & \multirow[b]{2}{*}{$\mathrm{p}$} & \multirow[b]{2}{*}{ LSD } \\
\hline & $\mathrm{F}$ & $\mathrm{p}$ & $\mathrm{H}$ & $\mathrm{p}$ & $\mathrm{F}$ & $\mathrm{p}$ & & & \\
\hline Número de espécies & & & 19,33 & $0,02 *$ & & & 16,31 & $0,00 * *$ & \\
\hline Número de peixes & & & 22,00 & $0,009 * *$ & 26,26 & $0,00 * *$ & & & $B>\underline{A C}$ \\
\hline Peso total & 19,00 & $0,00 * *$ & & & & & 22,22 & $0,00 * *$ & \\
\hline Riqueza & & & 8,66 & $0,46 \mathrm{NS}$ & 4,51 & $0,02 * *$ & & & $\mathrm{~B}>\underline{\mathrm{AC}}$ \\
\hline Diversidade (número) & & & 11,33 & $0,25 \mathrm{NS}$ & & & 3,78 & $0,15 \mathrm{NS}$ & \\
\hline Diversidade (peso) & & & 19,33 & $0,02 *$ & 7,46 & $0,00 * *$ & & & $\underline{\mathrm{BC}} \underline{\mathrm{CA}} \quad \mathrm{B}>\mathrm{A}$ \\
\hline Eqüitatividade & 4,38 & $0,00 * *$ & & & & & 0,50 & $0,77 \mathrm{NS}$ & \\
\hline
\end{tabular}

número de espécies e peixes, biomassa, riqueza e diversidade (peso) (tab. III). O grupo B, com similaridade de $64 \%$, é constituído por dois subgrupos, um agregando os meses de novembro e dezembro e o outro, os meses de janeiro a abril, ambos correspondendo ao período com as maiores capturas médias em número de espécies e peixes, biomassa e riqueza de espécies (tab. III). Unido no nível de similaridade de $68 \%$, o agrupamento $\mathrm{C}$ dos meses de maio e junho, com as capturas em número de espécies e peixes, biomassa, riqueza e diversidade (peso) não diferindo estatisticamente do grupo A, porém significativamente menores do que as do grupo B (tab. III). A diversidade (H' peso) foi significativamente maior no grupo B em comparação ao agrupamento A, no entanto não diferiu estatisticamente do grupo $\mathrm{C}$. Estes agrupamentos definidos no dendrograma (nível de $59 \%$ ) estão evidentes no MDS, com o estresse de 0,098 indicando que as distâncias gráficas entre os meses se aproximam das similaridades originais (fig. 3).

A análise de similaridade de percentagens (Simper) mostrou que $P$. harroweri, L. breviceps, $S$. rastrifer, Trinectes microphthalmus, Symphurus plagusia e L. laevigatus foram as espécies mais comuns $(69,3 \%)$ no grupo A definido pela Análise de Cluster (modo Q) (tab. IV). No grupo B, as espécies $P$. harroweri, L. breviceps, Menticirrhus americanus, $P$. brasiliensis, S. brasiliensis, S. rastrifer e L. laevigatus foram as mais representativas $(54,48 \%)$. Também foi possível observar que a dissimilaridade foi maior entre os grupos A e C $(55,29 \%)$, com os maiores contribuintes $S$. setapinnis, Selene vomer, C. nobilis, S. rastrifer, Peprilus paru e S. plagusia (53\% da dissimilaridade entre os dois grupos). Entre os grupos A e B as espécies mais discriminantes foram Caranx ruber, S. setapinnis, C. nobilis, Cynoscion microlepidotus, Isopisthus parvipinnis, L. breviceps, M. americanus, Menticirrhus littoralis e $S$. brasiliensis, responsáveis por $43 \%$ da dissimilaridade entre esses grupos. Contribuíram mais para a dissimilaridade existente entre os grupos B e C (46,18\%), C. nobilis, L. breviceps, M. americanus, $S$. brasiliensis, $S$. rastrifer e $P$. paru.

As vinte e sete espécies dominantes selecionadas apresentaram um padrão de ocorrência temporal. A
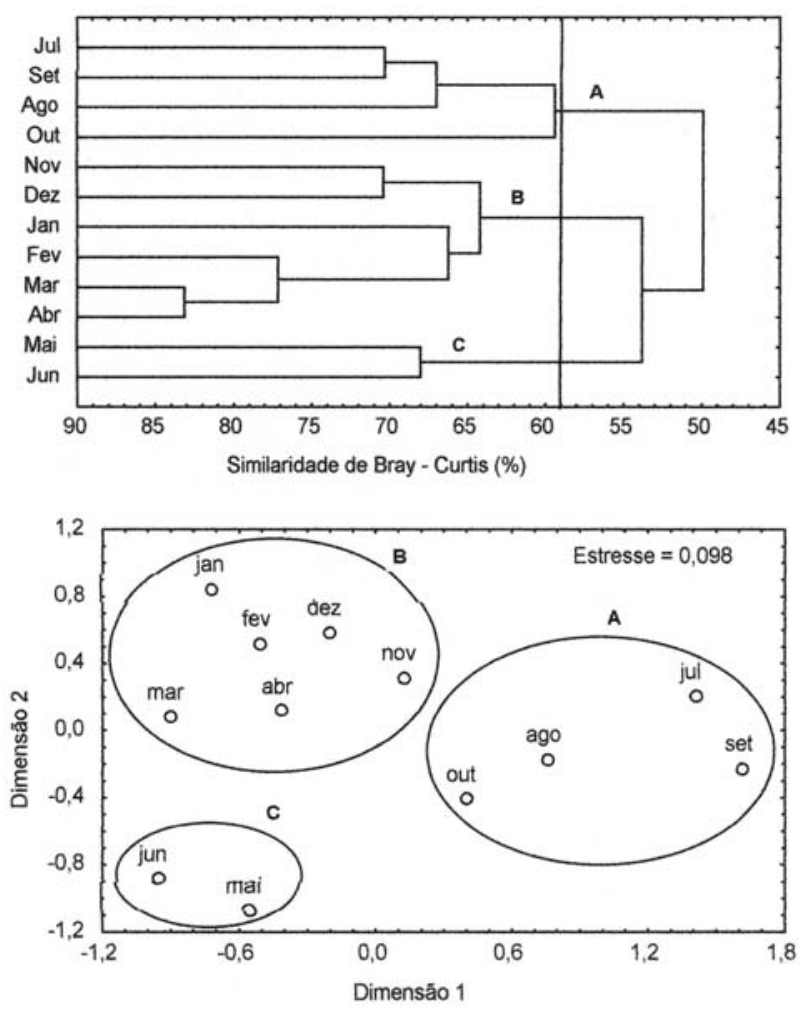

Fig. 3. Dendrograma e ordenação pelo método MDS baseados nos dados de densidade dos vinte e sete táxons principais, amostrados mensalmente, entre julho de 1998 e junho de 1999, no Balneário de Atami, Paraná. Grupos de espécies delineados no nível de 59\% de similaridade no dendrograma estão circundados no gráfico de ordenação. Estresse da ordenação MDS $=0,098$.

análise de Cluster (modo R) agrupou essas espécies em três grandes grupos com nível de similaridade de $58 \%$ (fig. 4). Foram unidas em um nível de similaridade de $59 \%$ (grupo A), as espécies $S$. rastrifer, L. breviceps, P. brasiliensis, Anisotremus surinamensis, C. ruber, $M$. americanus, S. brasiliensis e C. nobilis, todas presentes na área nos estágios juvenil e adulto. O grupo A é constituído por dois subgrupos, um formado pelas espécies $S$. rastrifer, L. breviceps, $P$. brasiliensis, as mais abundantes na área, presentes o ano inteiro e em maior quantidade entre o final da primavera e a metade do outono e outro formado pelas espécies $A$. surinamensis, C. ruber, M. americanus, S. brasiliensis 
Tabela IV. Contribuição percentual (\%) das espécies mais abundantes e constantes no infralitoral da praia Balneário de Atami, Paraná, para a similaridade dentro do grupo A (julho, agosto, setembro e outubro), do grupo B (novembro, dezembro, janeiro, fevereiro, março e abril) e do grupo $\mathrm{C}$ (maio e junho) e para a dissimilaridade entre esses grupos (A x B, A x C, B x C).

\begin{tabular}{|c|c|c|c|c|c|c|}
\hline Similaridade média dentro dos grupos $(\%)$ & $\begin{array}{c}\text { A } \\
63,75\end{array}$ & $\begin{array}{c}\text { B } \\
68,02\end{array}$ & Dissimilaridade média entre os grupos $(\%)$ & $\begin{array}{l}A \times B \\
48,35\end{array}$ & $\begin{array}{c}\mathrm{A} \times \mathrm{C} \\
55,29\end{array}$ & $\begin{array}{l}\text { B x C } \\
46,18\end{array}$ \\
\hline Pellona harroweri & 11,14 & 4,46 & Caranx ruber & 5,43 & & \\
\hline Larimus breviceps & 9,35 & 9,87 & Selene setapinnis & 5,25 & 8,38 & \\
\hline Menticirrhus americanus & & 5,97 & Selene vomer & & 5,28 & \\
\hline Paralonchurus brasiliensis & & 9,32 & Conodon nobilis & 6,94 & 8,46 & 4,53 \\
\hline Stellifer brasiliensis & & 8,52 & Cynoscion microlepidotus & 6,81 & & \\
\hline Stellifer rastrifer & 21,81 & 11,73 & Isopisthus parvipinnis & 5,59 & & \\
\hline Trinectes microphthalmus & 7,99 & & Larimus breviceps & 6,45 & & 5,67 \\
\hline Symphurus plagusia & 8,79 & & Menticirrhus americanus & 6,21 & & 5,31 \\
\hline \multirow[t]{5}{*}{ Lagocephalus laevigatus } & 10,17 & 4,61 & Menticirrhus littoralis & 4,97 & & \\
\hline & & & Stellifer brasiliensis & 7,67 & & 4,87 \\
\hline & & & Stellifer rastrifer & & 8,44 & 9,45 \\
\hline & & & Peprilus paru & & 5,52 & 6,11 \\
\hline & & & Symphurus plagusia & & 4,60 & \\
\hline
\end{tabular}
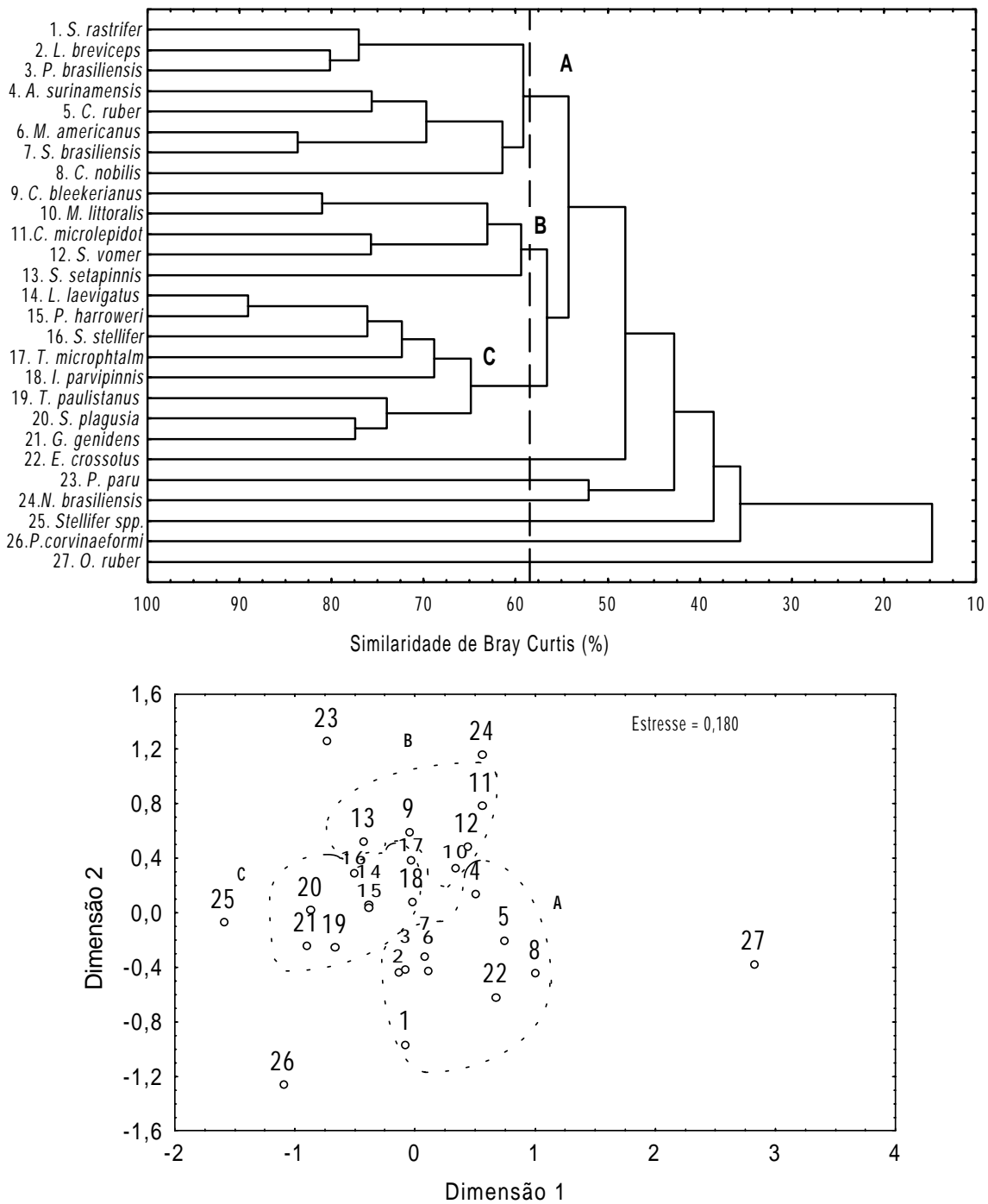

Fig. 4. Dendrograma e ordenação pelo método MDS, mostrando as similaridades entre os táxons mais abundantes baseadas em sua ocorrência ao longo dos doze meses de amostragem, entre julho de 1998 e junho de 1999, no Balneário de Atami, Paraná. Grupos de espécies delineados no nível de $58 \%$ de similaridade no dendrograma estão circundados no gráfico de ordenação. Estresse da ordenação MDS = 0,180. 
e C. nobilis, praticamente ausentes no inverno e primavera, com maior ocorrência entre o verão e o outono. O grupo B, apresentando similaridade de 59\%, foi caracterizado pelas capturas das espécies Chirocentrodon bleekerianus, $M$. littoralis, $C$. microlepidotus, S. vomer e S. setapinnis, as duas primeiras representadas por exemplares juvenis e adultos, enquanto que as outras somente por juvenis, presentes em geral em pequeno número em todas as estações do ano, com algumas ocorrências maiores e esporádicas, tanto no período de verão quanto no inverno, e menos freqüentes entre parte do inverno e parte da primavera. Agrupado no nível de similaridade de $65 \%$, o grupo C, formado por L. laevigatus, $P$. harroweri, Stellifer stellifer, T. microphthalmus, I. parvipinnis, Trinectes paulistanus, S. plagusia e Genidens genidens, presentes nas amostras nos estágios juvenil e adulto, à exceção de L. laevigatus, presente somente como juvenil, o qual está dividido em dois subgrupos, um unido no nível de similaridade de $69 \%$, comportando as espécies L. laevigatus, $P$. harroweri, S. stellifer, T. microphthalmus, I. parvipinnis, presentes em todas as estações do ano e com alguns picos de ocorrência no final da primavera, verão e inverno. Agrupado no nível de similaridade de $74 \%$, o segundo subgrupo, englobando as espécies $T$. paulistanus, S. plagusia e G. genidens, com um padrão de ocorrência similar ao subgrupo anterior, porém com freqüências mensais menores e ausência nos arrastos do final do outono ao início do inverno. Isolados destes três grupos, os táxons Etropus crossotus, $P$. paru, Narcine brasiliensis, Stellifer sp., Pomadasys corvinaeformis e $O$. ruber, ausentes em vários meses do ano e com picos esporádicos, exceção a $N$. brasiliensis. Os grupos definidos no nível de $58 \%$ no dendrograma são visualizados no MDS, com um estresse de 0,180 indicando que as similaridades não estão adequadamente representadas pelas distâncias no plano (fig. 4).

\section{DISCUSSÃO}

Durante o período amostrado, poucas espécies dominaram tanto em número quanto em peso. Esta pouca dominância também foi observada nas assembléias de peixes demersais da plataforma continental brasileira (FAgUndes NetTo \& GAELZER, 1991; Rossi-Wongtschowski \& Paes, 1993; Haimovici et al., 1994, 1996; Haimovici, 1998; Rocha \& RossiWongtschowski, 1998; Muto et al., 2000) e em estuários e baías da região sudeste-sul do Brasil (Pereira, 1994; Araújo et al., 1998; Chaves \& Bouchereau, 1999). Em parte, a dominância em número e peso foi exercida por um elenco diferente de espécies na praia Balneário Atami. As espécies S. setapinnis, O. ruber e S. brasiliensis, com grande contribuição em número, não tiveram uma participação significativa na biomassa em peso, tendo ocorrido o oposto $\operatorname{com} M$. americanus e G. genidens; este padrão parece estar refletindo os diferentes estágios em que foram capturados os exemplares, uma vez que algumas espécies utilizam a área apenas para recrutamento, enquanto outras a utilizam durante todo o ciclo de vida. Uma contribuição diferenciada para a estrutura em número e peso também foi observada em áreas costeiras expostas (Haimovici et al., 1996; Rocha \& RossiWongtschowski, 1998; Muto et al., 2000) e em outras áreas mais protegidas (ARAúso et al., 1998; CHAVES \& Bouchereau, 1999). As séries de dominância foram significativamente diferentes entre áreas, com apenas $S$. rastrifer e $O$. ruber importantes tanto no infralitoral do Balneário Atami quanto em áreas estuarinas do litoral do Paraná (Chaves \& Bouchereau, 1999). Em comparação com outros levantamentos na plataforma continental, apenas $P$. brasiliensis esteve entre as espécies mais abundantes no Atami e na costa do litoral de São Paulo (Rossi-Wongtschowski \& Paes, 1993; Rocha \& Rossi-Wongtschowski, 1998; Muto et al., 2000), não existindo nenhuma entre as mais abundantes comum ao Atami e às regiões da plataforma do Rio Grande do Sul (Haimovici et al., 1994, 1996).

A assembléia local estava constituída quase que exclusivamente por juvenis de espécies marinhas, com 17 espécies estuarinas, que estariam utilizando a área para alimentação e crescimento. Este uso de áreas costeiras rasas da plataforma continental em parte do ciclo de vida dos peixes demonstra a importância desse hábitat para as comunidades de peixes (LENANTON, 1982). Parecem estar utilizando a área tanto para desova quanto para o recrutamento, S. rastrifer, $P$. brasiliensis, I. parvipinnis, S. brasiliensis, S. stellifer, M. littoralis, $T$. paulistanus e $M$. americanus, enquanto que as outras 38 espécies estariam usando a região somente para os seus recrutas. As demais ocorreram esporadicamente sem qualquer padrão definido de uso da área. $\mathrm{O}$ curto tempo de residência da maioria dos componentes da ictiofauna local e o predomínio de migradores e visitantes ocasionais no Balneário Atami, que assim como as demais utilizam a área para alimentação, também foram observados nas comunidades de peixes demersais de estuários e baías (Pereira, 1994; Araúso et al., 1998) e da plataforma continental entre o Rio de Janeiro e Rio Grande do Sul (FAgundes Netto \& Gaelzer, 1991; Haimovici et al., 1994; Rocha \& Rossi-Wongtschowski, 1998). Muitas das formas migratórias presentes no infralitoral raso do Atami estiveram nas amostras da ictiofauna demersal do setor euhalino da Baía de Paranaguá, e em áreas estuarinas mais internas da baía de Guaratuba (Chaves \& Boucherau, 1999), no domínio interno da plataforma continental de São Paulo (Rocha \& RossiWongtschowski, 1998; Muto et al., 2000) e em menor escala na região costeira do Rio Grande do Sul (Pereira, 1994; Haimovici et al., 1994, 1996).

É aconselhável a padronização de métodos de amostragem que permitam minimizar as tendências associadas com as técnicas de coleta na comparação da composição e do padrão de abundância entre os diferentes ecossistemas. Contribuem para a subestimação da abundância peixes maiores e mais ativos, adultos agregados em cardumes, que evitam redes de arrasto, como as redes de porta e a exclusão de pequenos adultos e juvenis pela malha da rede (YoKlavich et al., 1991). Mesmo sendo diferentes as 
metodologias de coleta aplicadas na região sudestesul do Brasil, o que poderia estar contribuindo para as dissimilaridades observadas, uma maior similaridade ictiofaunística ocorre entre o infralitoral do Atami e as áreas costeiras de São Paulo e do Rio de Janeiro (ARAÚJo et al., 1998; Rocha \& Rossi-Wongtschowski, 1998; Muto et al., 2000) e até mesmo com o litoral de Pernambuco e Alagoas (SAntos et al., 1998). São grandes as diferenças na composição e abundância da ictiofauna demersal, ao compararmos os resultados do presente trabalho com os levantamentos realizados em áreas mais ao sul, algumas até mais próximas da praia de Atami (Haimovici et al., 1994, 1996; Pereira, 1994).

Esta diminuição na similaridade entre as latitudes está relacionada com a contribuição diferenciada de táxons de regiões tropicais, subtropicais e temperadas para a formação dessas assembléias de peixes. Entre as 70 espécies presentes na área estudada, 39 têm as suas distribuições desde as áreas tropicais até a região sudeste do Brasil, 22 da região tropical até a costa da Argentina, 6 espécies entre a região sudeste do Brasil e a Argentina e 2 espécies de Santa Catarina a Argentina (Figueiredo \& Menezes, 1978, 1980, 2000; Menezes \& Figueiredo, 1980, 1985). Espécies com distribuição limitada à região sudeste-sul da América do Sul, como Urophycis brasiliensis, Porichthys porosissimus, Trachinotus marginatus, $S$. brasiliensis, Umbrina canosai, Pseudopercis semifasciata, Oncopterus darwinii e Achirus lineatus (com exceção de $S$. brasiliensis, cuja distribuição vai da Bahia até São Paulo) (Menezes \& Figueiredo, 1980), abundantes na praia de Atami do final da primavera até a metade do outono, as demais espécies só apareceram em pequena quantidade, no estágio juvenil, praticamente somente no inverno. O inverno no litoral do Paraná parece não oferecer condições adequadas, pelo menos na área rasa logo após a zona de arrebentação, para a reprodução e a criação dessas espécies e de outras comumente associadas a águas mais frias (HAIMOVICI et al., 1994, 1996). Estes resultados revelam que a ictiofauna do local é de transição e mais similar a da região tropical de águas quentes, principalmente caraíbica, uma característica da ictiofauna do litoral do Paraná descrita anteriormente.

Nas amostras obtidas, foram importantes principalmente as espécies tropicais da família Sciaenidae, que contribuíram com cerca de $68 \%$ da captura total na área, constituindo-se a ictiofauna local em uma comunidade tropical de sciaenídeos, segundo Longhurst \& Pauly (1987). Na plataforma de São Sebastião, Ubatuba e entre Cabo de São Tomé e Torres, também predominaram os sciaenídeos tropicais (RochA \& Rossi-Wongtschowski, 1998; Muto et al., 2000), o que não ocorreu nas comunidades de peixes da plataforma de Santa Catarina e Rio Grande do Sul (Haimovici et al., 1994, 1996; Haimovici, 1998).

O infralitoral estudado é utilizado por uma ictiofauna composta principalmente por espécies sazonais que entram no local como juvenis ou, em menor quantidade, como adultos reprodutores. Os padrões de abundância das várias espécies, em especial das dominantes, parecem estar relacionados com os hábitos reprodutivos e subseqüente recrutamento na área. Uma maior abundância de juvenis entre o final da primavera até a metade do outono, o período de maior recrutamento, quando também é maior o número de adultos nos arrastos, seria responsável pelas maiores capturas em número e peso nessa fase do ano no infralitoral. Na região, uma maior atividade reprodutiva dos peixes na primavera e verão e a fase de recrutamento estendendo-se do verão até o outono também foram constatadas por Chaves \& Vendel (1997), Godefroid et al. (1997), Chaves \& Отto (1999). A diminuição observada nas capturas em número e peso entre o outono e a primavera pode ser atribuída à emigração dos residentes temporários, tanto juvenis quanto adultos, além das espécies que dominaram as capturas na área. Esta natureza transiente da assembléia de peixes, com os processos de imigração e emigração, na sua maioria de migradores oceânicos, em deslocamento para áreas de reprodução, alimentação e crescimento, foi observada em vários ambientes de águas rasas (LENANTON, 1982; YoKLAVICH et al., 1991; SZEDlmayer \& Able, 1996).

O maior número de espécies observado do final da primavera até a metade do outono, com uma assembléia menos rica em espécies no inverno e primavera no infralitoral do Atami, corresponde em parte ao anteriormente constatado em outras áreas demersais da Baía de Paranaguá e no canal de entrada da Lagoa dos Patos (Pereira, 1994).

\section{REFERÊNCIAS BIBLIOGRÁFICAS}

Araúso, F. G.; Cruz-Filho, A. G. et al. 1998. Estrutura da comunidade de peixes demersais da Baía de Sepetiba, RJ. Revista Brasileira de Biologia, Rio de Janeiro, 58(3):417-430.

Bergstad, O. A.; Buelland, O. \& Gordon, J. D. M. 1999. Fish communities on the slope of the eastern Norwergian Sea. Sarsia, Bergen, 84:67-78.

Blaber, S. J. M.; Brewer, D. T. \& Salini, J. P. 1989. Species composition and biomasses of fishes in different habitats of a tropical northern Australian estuary: their occurrence in the adjoining sea and estuarine dependence. Estuarine Coastal and Shelf Science, London, 29:509-531.

Chaves, P. T. C. \& Bouchereau, J-L. 1999. Biodiversité et dynamique des peuplements ichtyiques de la mangrove de Guaratuba, Brézil. Oceanologica Acta, Paris, 22(3):353364

Chaves, P. T. C. \& CorrêA, M. F. M. 1998. Composição ictiofaunística da área de manguezal da Baía de Guaratuba, Paraná, Brasil. Revista Brasileira de Zoologia, Curitiba, 15(1): 195-202.

Chaves, P. T. C. \& Отто, G. 1999. The mangrove as a temporary habitat for fish: the Eucinostomus species at Guaratuba Bay, Brazil (2552'S; 48³9'W). Brazilian Archives of Biology and Technology, Curitiba, 42(1):6168.

Chaves, P. T. C. \& Vendel, A. L. 1997. Indicadores reprodutivos das espécies Citharichthys bleeker (Teleostei, Pleuronectiformes) na Baía de Guaratuba, Paraná, Brasil. Revista Brasileira de Zoologia, Curitiba, 14(1):73-79.

Clarke, K. R. \&. Warwick, R. W. 1994. Change in marine communities: an aproach to statistical analysis and interpretation. Bournemouth, Bourne. 859 p.

Conover, W. J. 1990. Practical nonparametric statistics. New Jersey, John Willey \& Sons. 584p.

Fagundes Netto, E. B. \& Gaelzer, L. R. 1991. Associações de peixes bentônicos e demersais na região do Cabo Frio, RJ, Brasil. Nerítica, Curitiba, 6(1/2):139-156. 
Figueiredo, J. L. \& Menezes, N. 1978. Manual de peixes marinhos do sudeste do Brasil. II. Teleostei (1). São Paulo, Museu de Zoologia, USP. 110p.

1980. Manual de peixes marinhos do sudeste do Brasil. III. Teleostei (2). São Paulo, Museu de Zoologia, USP. $90 \mathrm{p}$

2000. Manual de peixes marinhos do sudeste do Brasil. VI. Teleostei (5). São Paulo, Museu de Zoologia, USP. $116 \mathrm{p}$.

Gauch, H. G., JR. 1982. Multivariate analysis in community ecology. London, Cambridge University. 298 p.

Godefroid, R. S.; Hofstaetter, M. \&. Spach, H. L. 1997. Structure of the fish assemblage in the surf zone beach at Pontal do Sul, Paraná. Nerítica, Pontal do Sul, 11:77-93.

Haimovici, M. 1998. Present state and perspectives for the southern Brazil shelf demersal fisheries. Fish Management of Ecology, Oxford, 5:277-289.

Haimovici, M.; Martins, A. S. et al. 1994. Demersal bony fish of the outer shelf and upper slope of the southern Brazil subtropical convergence ecosystem. Marine EcologyProgress Series, Oldendorf, 108:59-77

Haimovici, M.; Martins, A. S. \& Vieira, P. C. 1996. Distribuição e abundância de peixes teleósteos demersais sobre a plataforma continental do sul do Brasil. Revista Brasileira de Biologia, Rio de Janeiro, 56(1):27-50.

JACKSON, G. \& Jones, G. K. 1999. Spatial and temporal variation in nearshore fish and macroinvertebrate assemblage from a temperate Australian estuary over a decade. Marine Ecology-Progress Series, Oldendorf, 182:253-268

Kallianotis, A.; Sophronidis, K. et al. 2000. Demersal fish and megafaunal assemblages on the Cretan continental shelf and slope (NE Mediterranean): Seasonal variation in species density, biomass and diversity. Progress in Oceanography, Oxon, 46:429-455.

Lenanton, R. C. J. 1982. Alternative non-estuarine nursery habitats for some commercially and recreationally important fish species of south western Australia. Australian Journal of Marine and Freshwater Research, Melbourne, 33:881-900.

Longhurst, A. R. \& Pauly, D. 1987. Ecology of tropical oceans. San Diego, Academic. 407p
Ludwig, J. A. \& Reynolds, J. F. 1988. Statistical ecology. New York, John Wiley \& Sons. 337p.

Menezes, N. \& Figueiredo, J. L. 1980. Manual de peixes marinhos do sudeste do Brasil. IV. Teleostei (3). São Paulo, Museu de Zoologia, USP. 90p.

1985. Manual de peixes marinhos do sudeste do Brasil. V. Teleostei (4). São Paulo, Museu de Zoologia, USP. 90p.

Muto, E. Y.; Soares, L. S. H. \& Rossi-Wongtschowski, C. L. D. B. 2000. Demersal fish assemblages of São Sebastião, southeastern Brazil: structure and environmental conditioning factors (summer 1994). Revista Brasileira de Oceanografia, São Paulo, 48(1):9-2.

Pereira, L. E. 1994. Variação diurna e sazonal dos peixes demersais na barra do estuário da Lagos dos Patos, RS Atlântica, Rio Grande, 16:5-21.

Pielou, E. C. 1969. The measurement of diversity in different types of biological colections. Journal of Theoretical Biology, London, 13:131-144.

Rocha, G. R. A. \& Rossi-Wongtschowski, C. L. D. B. 1998. Demersal fish community on the inner shelf of Ubatuba, southeastern Brazil. Revista Brasileira de Oceanografia São Paulo, 46(2):93-109.

Rossi-Wongtschowski, C. L. D. B. \& Paes, E. T. 1993. Padrões espaciais e temporais da comunidade de peixes demersais do litoral norte do Estado de São Paulo, Ubatuba, Brasil. Boletim do Instituto Oceanográfico, São Paulo, 10:169188 .

Santos, M. C. F.; Freitas, A. E. T. \& Silva, M. M. 1998. Composição da ictiofauna acompanhante da pesca de camarão em Tamandaré/PE e Pontal do Peba/AL. Boletim Técnico Científico do CEPENE, Tamandaré, 1(1):4759.

Sokal, R. R. \& Rohlf, F. J. 1995. Biometry. New York, W. H. Freeman. $859 \mathrm{p}$.

Szedlmayer, S. T. \& Able, K. W. 1996. Patterns of seasonal availability and habitat use by fishes and decapod crustaceans in a southern New Jersey estuary. Estuaries, Lawrence, 19(3):697-709.

Yoklavich, M. M.; Calliet, G. M. et al. 1991. Temporal and spatial patterns in abundance and diversity of fish assemblages in Elkhorn Slough, California. Estuaries, Lawrence, 14(4):465-480. 